\title{
POTENTIAL OF A DRY ROTATING-DISK MAGNETIC SEPARATOR
}

\section{J. GERHOLD}

Technical University, A-8010 Graz, Austria

(Received December 18, 1991)

\begin{abstract}
Selectivity is a severe problem in dry magnetic separation. Dry rotating-disk magnetic separator utilizes radial magnetic forces that compete against centrifugal mass forces. Genuine equilibrium of forces, which is compulsory for high selectivity can thus be achieved at a high level of magnetic force. The potential of an iron-pole separator for intergrown ferromagnetics, as well as of a superconducting system for paramagnetic materials is discussed.
\end{abstract}

\section{INTRODUCTION}

Techniques of dry magnetic separation are often required for the treatment of finely grained materials. Flow-through separators can offer a simple solution for a total beneficiation process. However, magnetic separation should be selective. Random feeding conditions, as well as diferent particle sizes, must be tolerated within reasonable limits. moreover, selectivity is required in separation of mixtures where magnetic properties of particles differ only slightly.

One possible way towards selectivity is a multiple-pass separation, e.g. in induced magnetic roll separator. The feed, however, must be classified in advance according to particle size, which is a costly procedure. Throughput is limited because the feed must be presented as a monolayer.

The key to truly selective separation has been known for several decades [1]. Within the separation space, genuine stable or neutral equilibria between magnetic and competing forces must be maintained. Using gravity for the latter anadynamic or isodynamic magnetic fields are required. Katadynamic fields, as used in induced magnetic roll separators, or other common magnetic separators are, in principle, non-selective: magnetic force increases continuously when a particle is moving in the field, so the equilibrium with competing forces is impossible to achieve. 
Selectivity under equilibrium conditions is well established in Frantz isodynamic separator which is an analytical laboratory device. Unfortunately, Frantz isodynamic separator cannot be easily scaled-up to a production size machine. Competing mass forces in the gravity range are low and the feed must thus be presented in a well-dispersed form. Otherwise, perturbative interaction among the particles could impede the selective separation. A narrow air gap cannot be enlarged as a result of limitation of the iron pole saturation, and thus the capacity of the machine is low.

Dry rotating-disk magnetic separator (DRDS) was thought to be a way how to overcome shortcomings of the Frantz separator and still to retain the selectivity benefits. Instead of gravity, centrifugal mass forces, which can be easily adjusted, are used to compete with radial magnetic forces. Again, equilibrium conditions can be maintained in annular separation space.

\section{DRDS WORKING SCHEME}

DRDS, as shown in Figure 1 consists of a horizontal rotating disk, of a magnet system that generates magnetic field in which the forces act radially inwards over appropriate radial distance, and of means for feeding and discharging a material to be separated.

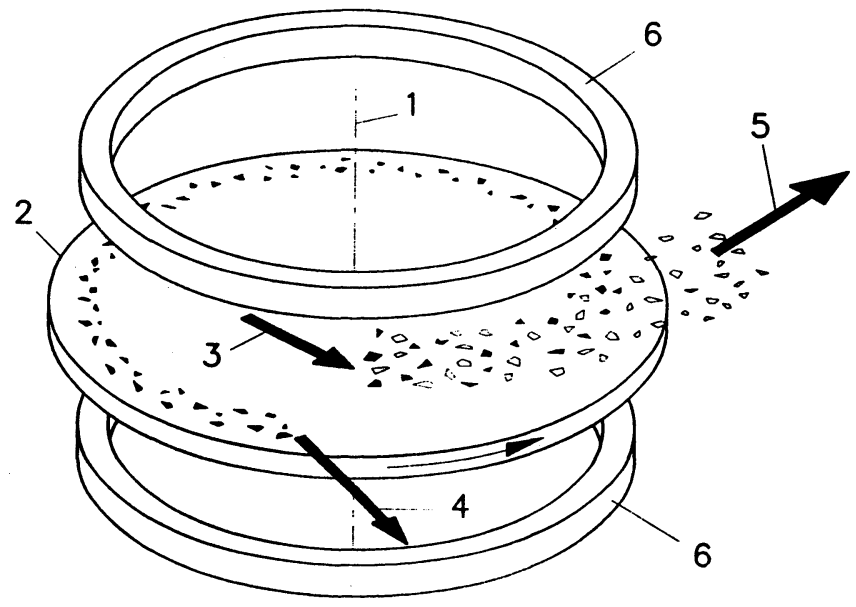

FIGURE 1 Scheme of DRDS operation.

1-axis of rotation, 2-rotating disk, 3-feed, 4-mags discharge, 5tails discharge, 6-split coil magnet 
When the mixture is fed onto the disk, the particles are accelerated by friction to angular rotation. The Froude number can be adjusted, for instance, between 5 to 10 times the gravity, which is appropriate for particles in the 50 micrometer to 1 $\mathrm{mm}$ size range. Weak magnetics are deflected to the outside and are collected in Tails collector. Stronger magnetics are deflected inwards and captured in a form of a rotating ring. These magnetic particles are ultimately discharged just before they reach again the feed region, for instance by means of a strong exhauster. There is no discharge of the middlings.

The DRDS is a combination of deflection and capture modes of separation. Various designs have been investigated, both theoretically, and on pilot-plant scale. Each version requires optimum profile of magnetic force. Such a magnetic field can be induced with properly shaped circular iron poles, adequate for separation of intergrown ferromagnetic grains. Superconducting solenoid magnets are required for separation of paramagnetic materials.

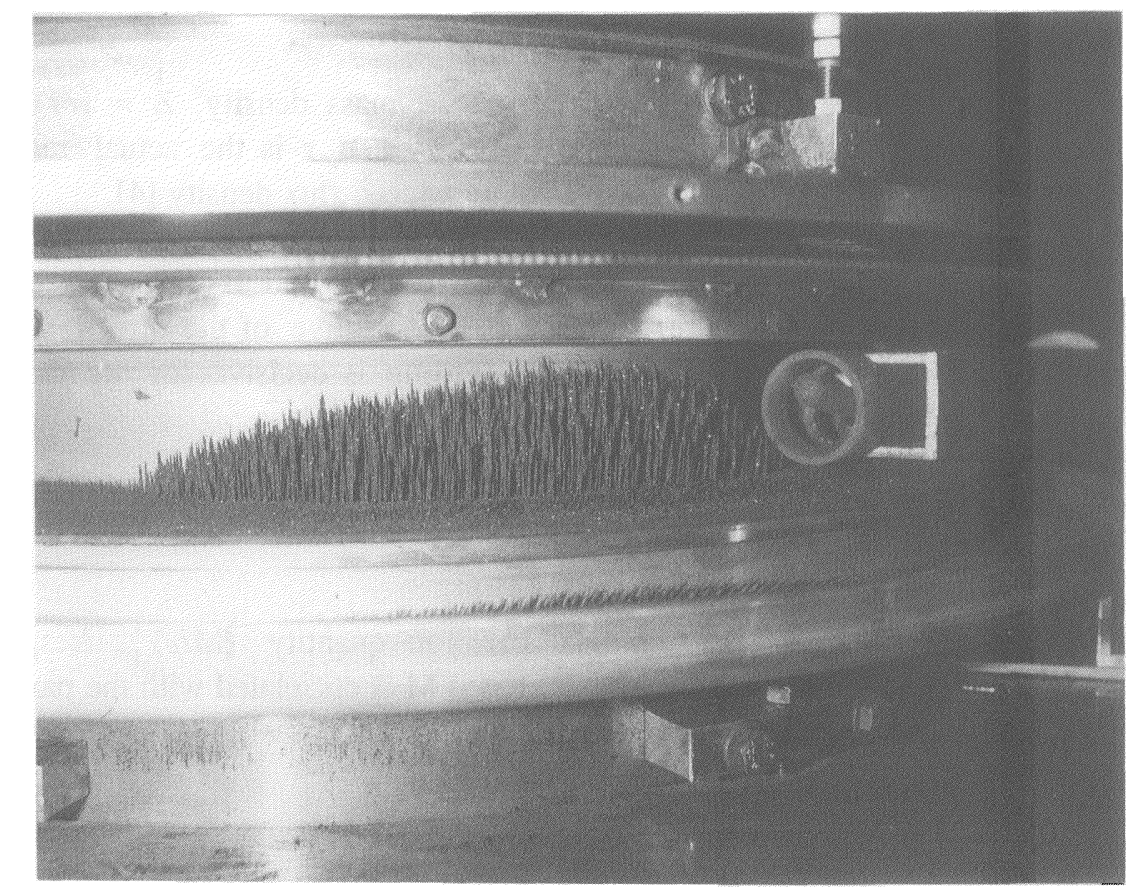

FIGURE 2 Agglomerated ferromagnetic needles in DRDS

Apart from optimum profile of the force field, direction of the magnetic flux lines is decisive. Flux lines can be either parallel with the force, or perpendicular to it [2]. The latter configuration has been found to be considerably more powerful. The captured magnetic fraction, which consists of magnetic dipoles, moves and groups in tandem when finally captured. These tandems are mutually repulsive so that 
interference with weak magnetics is limited [3], and the final discharge is easy. Figure 2 indicates the captured ferromagnetics showing individual needles.

\section{THEORETICAL ASSESSMENT}

The first and rough assessment can be derived from the separation forces acting onto individual particles. The mass forces are:

$$
\mathrm{F}_{\mathrm{z}}=\mathrm{V} \rho \mathrm{Z}
$$

and the competing magnetic forces can be written as:

$$
F_{M}=V M \operatorname{grad} B
$$

where $V$ is the particle volume, $\rho$ is the particle mass density, $Z=\omega^{2} r$ is the Froude number, where $\omega$ is the angular velocity and $r$ is the actual radius of rotation, $\mathbf{M}$ is the particle magnetization, $B$ is the induced flux density [4].

Since the particles must move freely in the separator, $\mathbf{M}$ and $\mathbf{B}$ are assumed to be parallel so that $F_{M}$ has the direction of $\operatorname{grad} B$, irrespective of the direction of the particular flux line. Consequently, the $|\mathrm{B}|$ - profile is decisive for the magnetic force profile [5]. Whenever the force equilibrium is achieved,

$$
\mathrm{F}_{\mathrm{M}} / \mathrm{F}_{\mathrm{z}}=1
$$

This relation formally defines the nominal partition quantity $(M / \rho)_{p}$, irrespective of particle size. The separation is selective only if $\mathbf{M}$ is correlated with the partition quantity in an univocal manner. There are two limiting cases. Ideal paramagnetics have a constant mass magnetic susceptibility $\chi$. Then,

$$
\mu_{\mathrm{o}} \mathrm{M}_{\mathrm{para}}=\chi \rho \mathrm{B}
$$

and

$$
\mathrm{F}_{\mathrm{M}} / \mathrm{F}_{\mathrm{z}}=\frac{\chi \mathrm{Bgrad} \mathrm{B}}{\mu_{\mathrm{O}} \mathrm{Z}}
$$

Saturated ferromagnetics that have constant magnetization $\mathbf{M}_{\mathbf{s}}$, so that: 


$$
\mathrm{F}_{\mathbf{M}} / \mathrm{F}_{\mathbf{Z}}=\frac{(\beta / \rho) \mathrm{M}_{\mathrm{s}} \operatorname{grad} \mathrm{B}}{\mathrm{Z}}
$$

can also be separated selectively, i.e. according to different ferromagnetic volume constant $\beta$ of the particles. Selectivity is considerably more questionable with minerals where the magnetization is nonlinear [4].

The aforementioned simple formulae neglect the demagnetization phenomena. This can be approximately correct in practice, up to the volume magnetic susceptibility somewhat below unity. In the ferromagnetic domain, this condition will require a not too low background flux density, because

$$
\chi \rho=\mu_{0} M_{s} / B
$$

However, real conditions in DRDS are more complicated. there is a relative motion of particles across the disk due to friction, and particle interactions are also present. To prevent interactions in an industrial machine where the feed is not presented as a monolayer, the particles must de dispersed within appropriate separation space. Annular rotating channel has been found the most suitable. Magnetic forces must act across the entire channel to capture all particles.

The particle motion has been numerically calculated for individual particles only. This analysis revealed some ideas about the potential performance of different modes of operation. Particle interactions were excluded from the numerical treatment and have been estimated in general terms.

\section{$\underline{\text { Individual Particles }}$}

Movement of individual particles has been investigated in detail for three principal versions of DRDS. (The background of calculations is given elsewhere [6].)

\section{i. Anadynamic magnetic force wall with the feed point inside}

ii. Isodynamic force profile, i.e. M grad B constant across the separation channel.

iii. Genuine neutral equilibrium force profile, i.e. $M$ grad B - r.

i. The force wall is shown in Figure 3. Any particle that touches the disk, as shown in Fig. 1, is accelerated towards the outside. The magnetics are impeded by the force wall and deflected; some reflections may occur. Particles with the magnetic susceptibility smaller than critical magnetic susceptibility may escape. Particles with susceptibility greater than the critical value are captured as a free rotating ring, i.e. in a hybrid mode. Typical particle trajectories for different arbitrary magnetic susceptibilities can also be seen in Fig. 3. By varying the feed point as 
well as friction, theoretical value of dispersion of separation around nominal partition susceptibility $x_{p}$ can be determined. It is found to be less than $0.2 x_{p}[6]$.

ii. The isodynamic force profile has been used with U-shaped rotating channel, as shown in Figure 4. There is a well- defined active channel cross section, i.e. radial distance $\Delta r$ and height $\Delta \mathrm{h}$. The captured magnetic particles settle on the inner wall of the channel. Reflections should be prevented. The feed point must be placed outside the separation channel. This appeared more convenient in view of practical magnet system. Typical trajectories are shown in Figure 5. Dispersion of the separation has been found to be considerably reduced, i.e. $\pm 0.05 x_{p}$, notwithstanding the slightly quasikatadynamic force concurrence [7]. This mode has been investigated in more detail, particularly with iron poles.

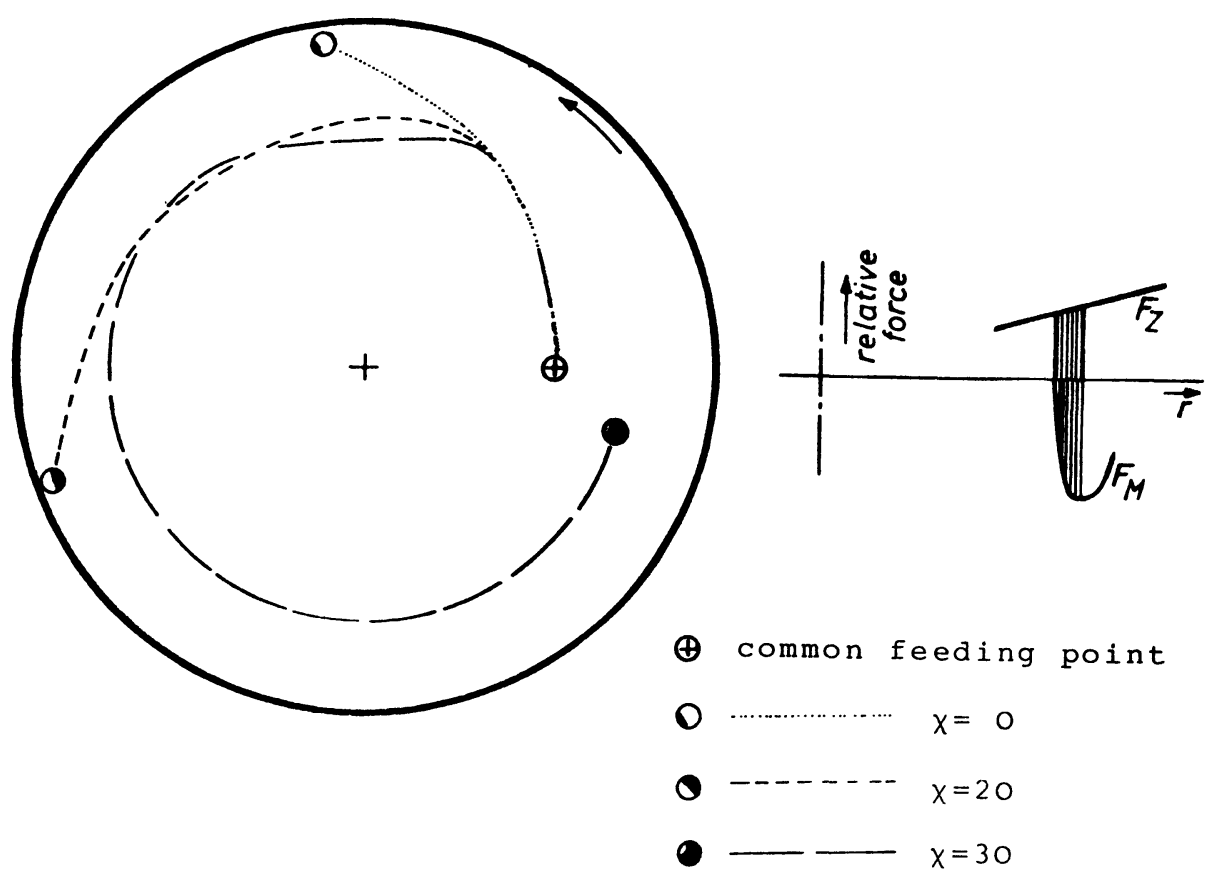

FIGURE 3 Anadynamic force wall $F_{M}$ with competing mass forces $F_{2}$ in DRDS; typical particle trajectories.

iii. From the first principles, a genuine neutral equilibrium force profile was not found to be superior to the version described above. However, if the feed is adjusted tangentially in such a way that there is no relative motion at the moment when particles touch the disk, the dispersion vanishes completely [7]. The corresponding partition curve is an ideal step function just at $\chi_{p}$. The magnetic par- 
ticles can be captured again in the vicinity of the inner wall of the channel, but the free rotating ring may also be of interest. This is indicated in Figure 6. In order to achieve this hybrid capture mode, the magnetic force must decrease rapidly just inside the inner wall of the channel. This can be very easily achieved with a superconducting solenoid. Consequently, this version has been investigated primarily having in view a superconducting separator for paramagnetic materials.

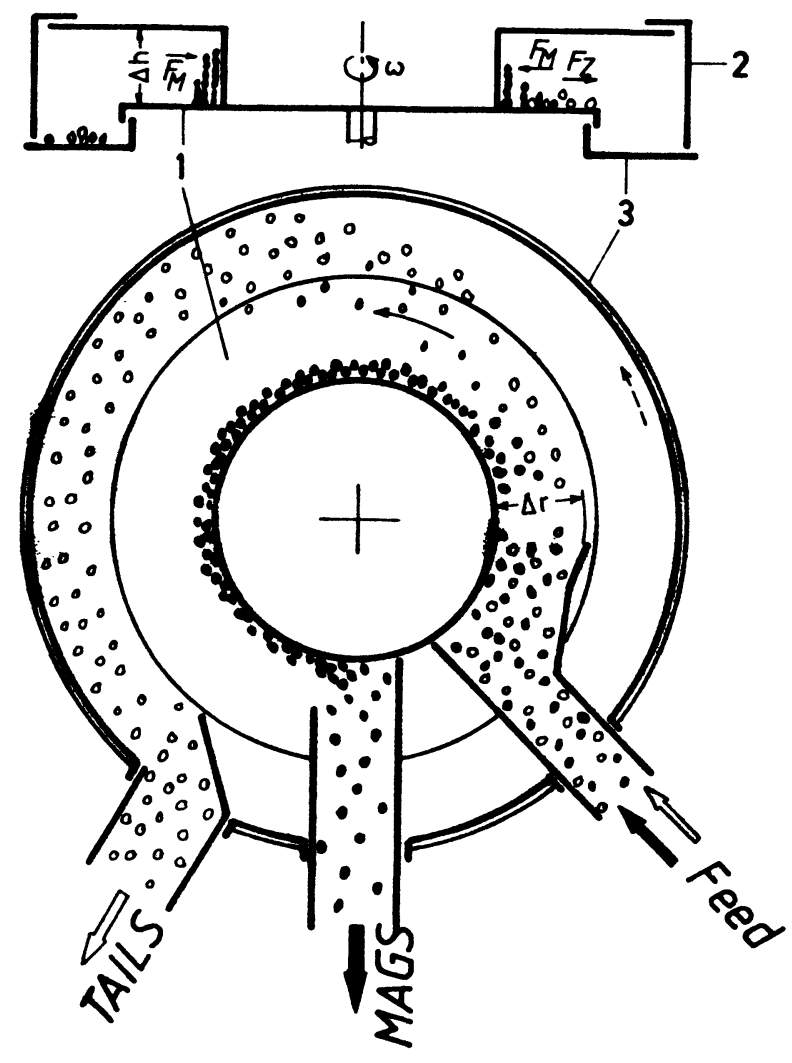

FIGURE 4 DRDS with U-shaped rotating channel; isodynamic mode.

1-rotating channel, 2-fixed tails apron, 3-slowly rotating tail collector

\section{$\underline{\text { Perturbative Effects }}$}

There are perturbations in real DRDS where the feed is not present in the form of a monolayer. To illustrate this aspect, Figure 7 shows a cut-away view of isodynamic separator EPMS 2 equipped with iron poles [8]. THe U-shaped channel has a mean radius $\overline{\mathrm{r}}=0.205 \mathrm{~m}$ and rotates between the iron poles of the 
magnet. The feed arrives via a rotating spiral feeder into the channel and is whirled up when touching it.

Acceleration up to full angular velocity takes about a quarter of revolution and is mainly due to friction. However, the air drag affects particularly light and very small particles. The air stream should therefore be adjusted carefully for every particular feed. This can be done by means of air entrance windows in the fixed tails apron. This apron closes the channel outside. The air that enters the channel is discharged together with the magnetic fraction.
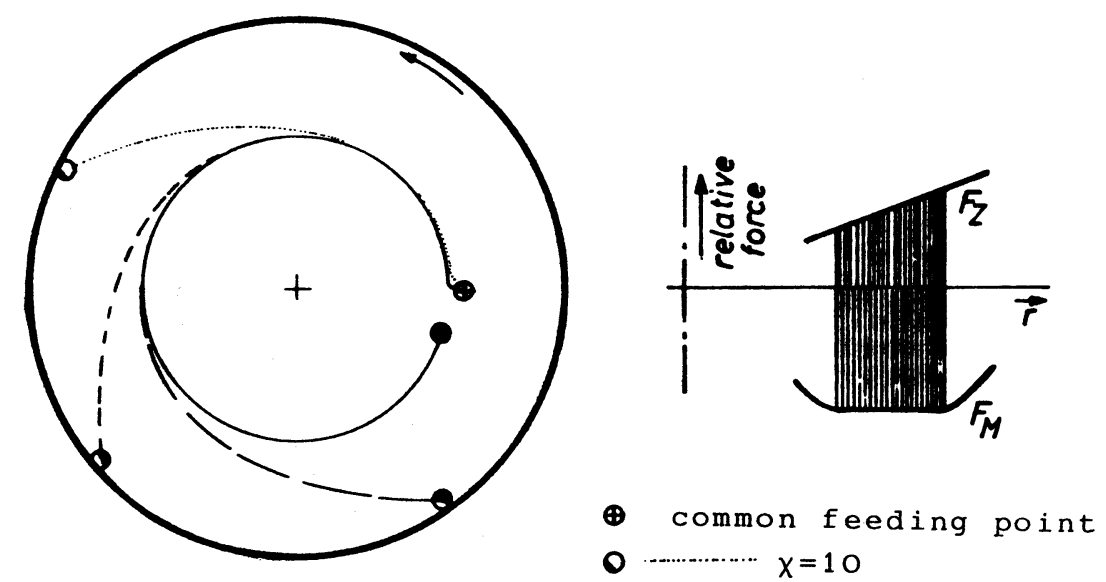

$0-\cdots-x=20$

$-x=30$

$x=40$

FIGURE 5 Isodynamic magnetic forces $F_{M}$ with competing mass forces $F_{Z}$ in DRDS; typical particle trajectories

Essentially, separation takes place during the first quarter of the first revolution; this corresponds to the length of approximately $0.5 \mathrm{~m}$. The whirled feed fills the channel in the form of a cloud. The volumetric filling factor $\epsilon=1 \%$ seems a reasonable assumption to ensure an unimpeded movement of individual particles [9]. Thus, the volumetric throughput $\mathrm{V}$ is given as:

$$
\dot{\mathrm{V}}=\zeta \Delta \mathrm{r} \Delta \mathrm{h} \omega \mathbf{r}
$$

i.e. $0.11 \mathrm{~m} 3 / \mathrm{s}$ for the EPMS2 channel at the force level of 10 times the gravity. The corresponding mass capacity is in the range of 1 to 2 tonnes per hour, depending on the mass density of the feed. 

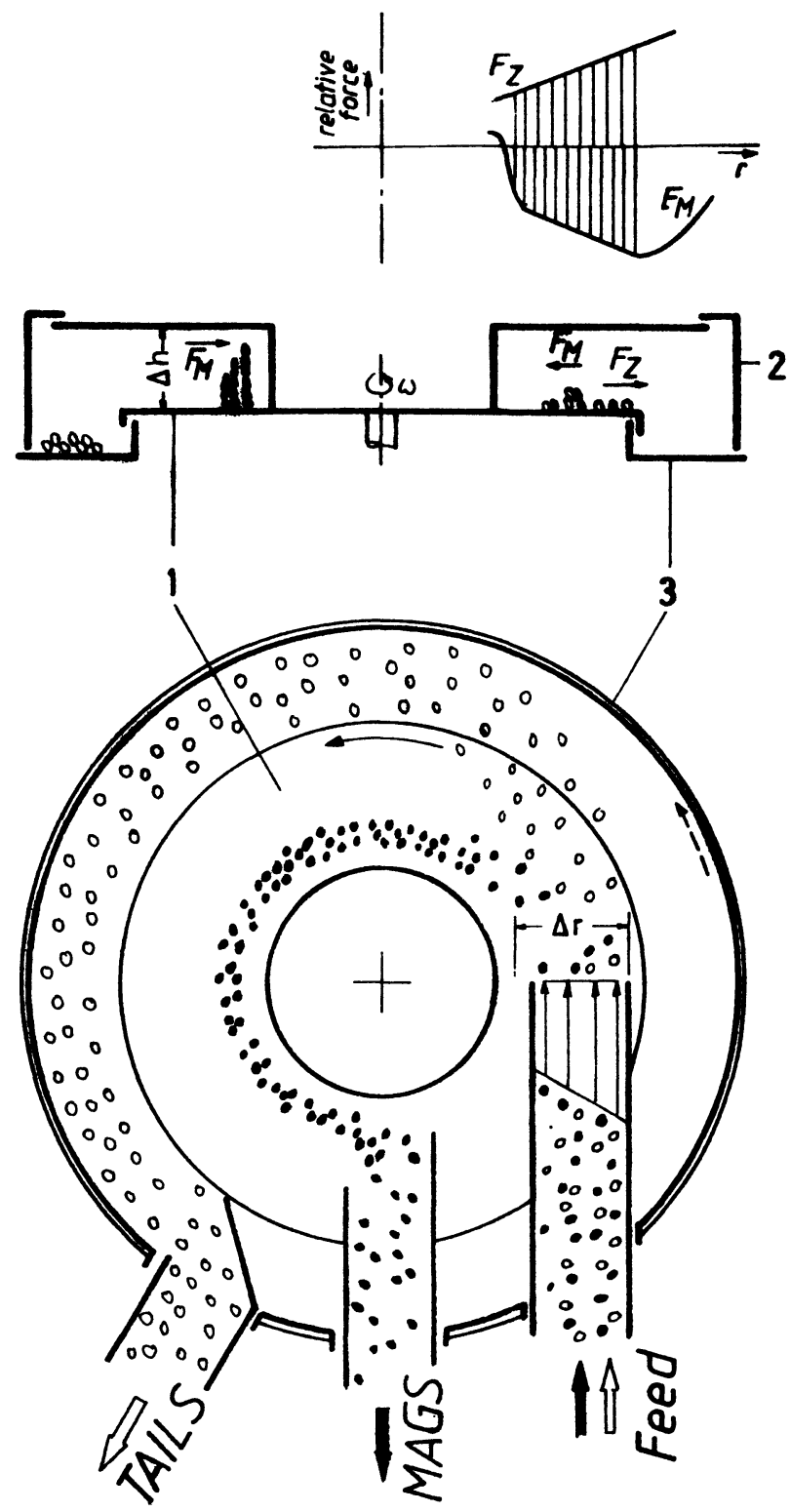

FIGURE 6 DRDS with U-shaped rotating channel in hybrid mode; genuine neutral equilibrium between magnetic forces $F_{M}$ and competing mass forces $F_{Z}$. 1-rotating channel, 2-tails apron, 3-slowly rotating tails collector

A suitable height of the channel is clearly required. Unfortunately, there is no possibility to obtain parallel magnetic forces. Strictly radial forces can only be 
maintained within the symmetry plane between the poles. This is a substantial phenomenon for nonuniform fields and can be seen from magnetostatic Maxwell equations [10]. Figure 8 illustrates the measured force vectors across the EPMS2 channel [11].

On the other hand, interparticle perturbative forces can be suppressed during the whirling-up process. Electrostatic, van der Waals and capillary moisture interactions are probably rather weak, but magnetic agglomeration can still have a detrimental influence. The cleaning of the captured magnetic fraction has been found difficult in DRDS, regardless of the weak coupling energy. Although the magnetic dipolar attraction is short-ranged, magnetic agglomeration can pose a severe problem with fine ferromagnetic materials, but can be rather ineffectual with paramagnetics in the superconducting version $[3,12]$.

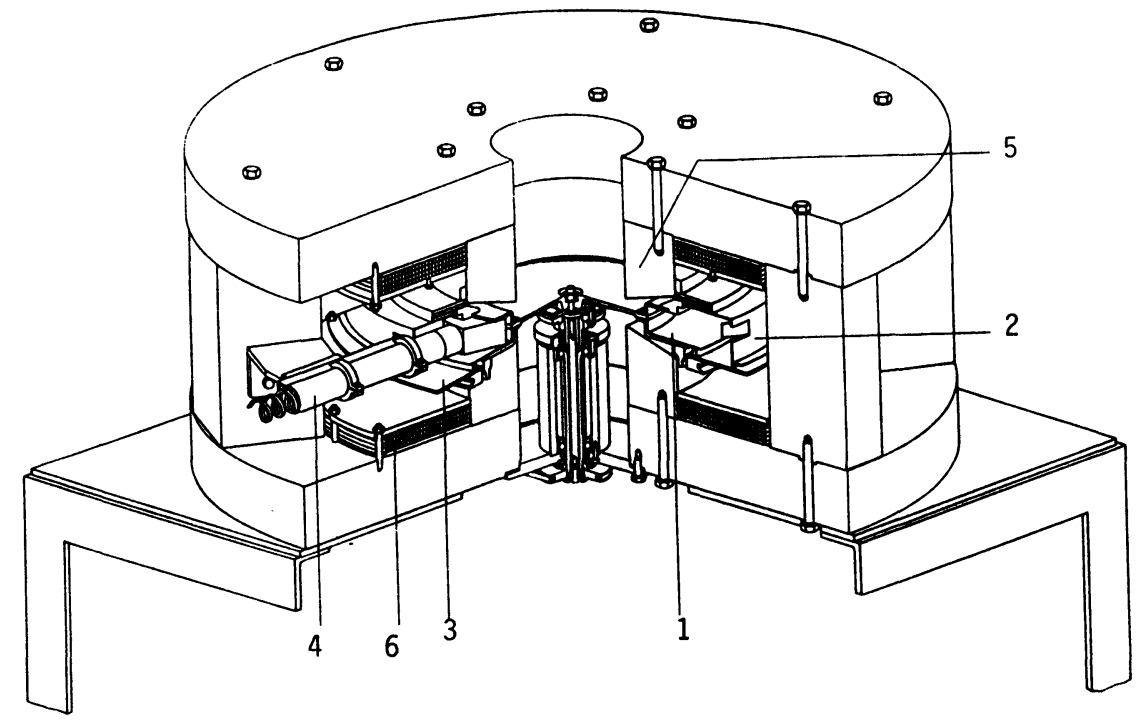

FIGURE 7 Iron-pole DRDS "EPMS2".

1-rotating channel, 2-tails apron, 3-tails collector, 4-rotating spiral feeder, 5-pole, 6-exciting coil.

\section{DRDS EXPERIMENTS}

To get a better insight into the process and its efficiency, several versions of the separator have been tested. The most relevant data were obtained with machines equipped with iron poles where the shape of the poles and of the channel and auxiliaries could be easily adapted. Cost of these machines is within reasonable limits. 
Separators with iron poles were found suitable for separation of intergrown ferromagnetics. Details of the tests have been published recently [12]. A typical grade-recovery diagram is shown in Figure 9.

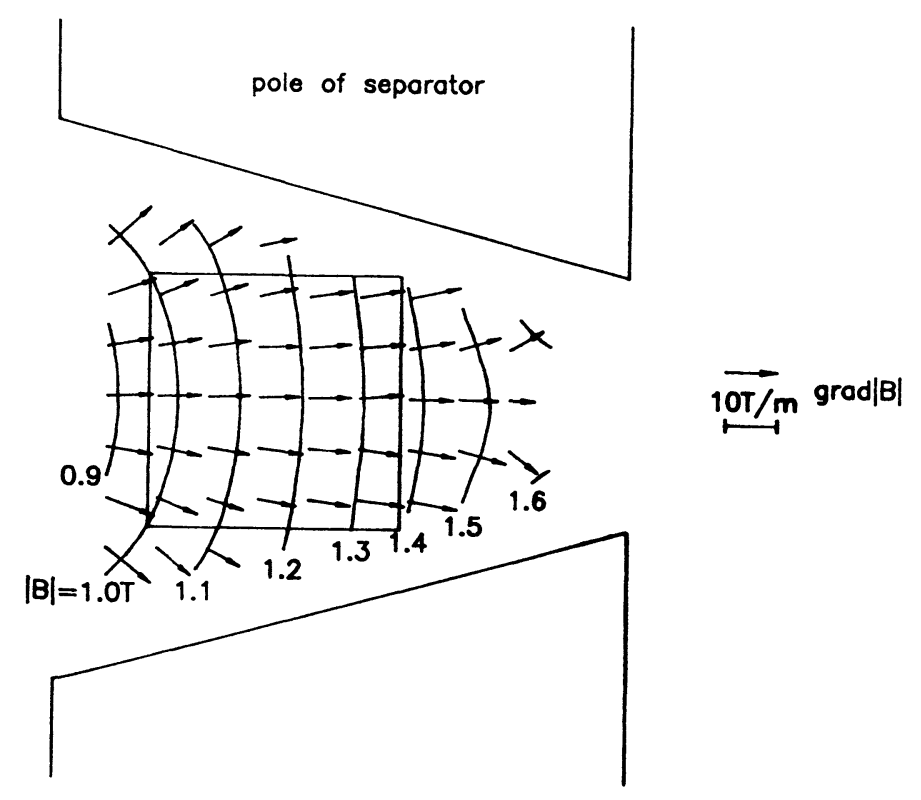

FIGURE 8 Measured magnetic force vectors across the separation channel of EPMS2

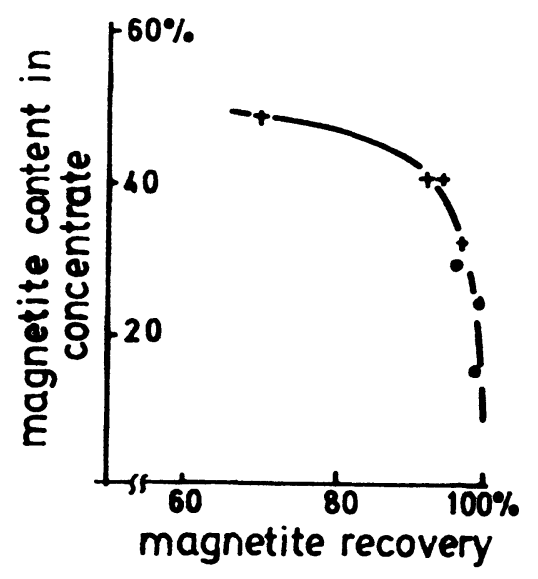

FIGURE 9 Grade versus recovery in EPMS2; feed $+50 \mu \mathrm{m}-3 \mathrm{~mm}$

However, the grade-recovery evaluation was found to be insufficient for assessment of further development. Therefore, the troublesome task of 
establishing partition curves has been undertaken. A special procedure had to be developed for magnetic classification of the ferromagnetics. This is described in detail in [12].

Figure 10 gives a typical partition curve found with natural mineral feed containing magnetite and gangue minerals such as hematite, quartz, pyrite etc. The separation quantity is $\beta / \rho$, according to eq.(6), since magnetite is strictly saturated; the minimum magnetic field in the channel being $0.9 \mathrm{~T}$.

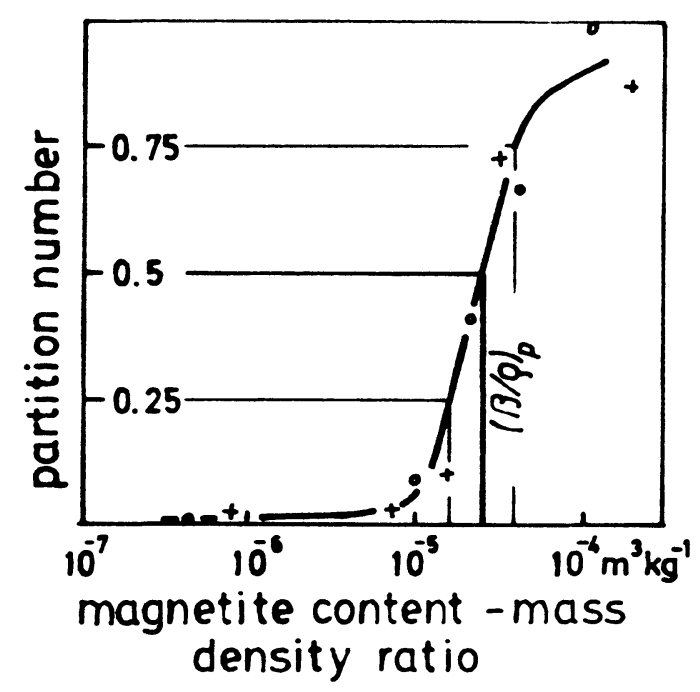

FIGURE 10 Partition curve of EPMS2, feed $+50 \mu \mathrm{m}-3 \mathrm{~mm}$

No distinct influence of particle size in the range $+50 \mu \mathrm{m}-1 \mathrm{~mm}$ has been found. Feed classification is thus not necessary. However, relative dispersion of separation is close to 1 , i.e. more than an order of magnitude higher than a theoretical prediction from simple calculations of particle trajectories. This figure may indicate the role of adverse perturbations acting in a real process.

Similarly dispersed partition curves have been found in approximately neutral equilibrium hybrid mode iii. Hitherto no attempts have been made to adjust the feed tangentially; certain degree of dispersion had thus to be expected. The main advantage is a much higher content of magnetite in the feed which can be treated reasonably easily [12]. Further optimization is clearly necessary of this promising mode. One option can be effective simplification of the process by means of an all-rotating channel [13]. The fixed apron can be simply replaced by a moving flat belt which is used simultaneously to drive the channel. Perturbative air drag can be 
drastically reduced. Slowly rotating tails collector can thus be be eliminated, too. A small scaled-up machine is under construction at the Technical University of Graz.

\section{SUPERCONDUCTING DRDS}

High flux density in superconducting magnets is attractive particularly for separation of paramagnetic minerals. It is known that some minerals exhibit a typical, approximately field-independent magnetic susceptibility at high fields, irrespective of the origin of the mineral [4]. On the other hand, weak magnetic agglomeration can result in improved selectivity [3].

The initial improved magnet configuration to be used for DRDS is a split-coil system with separation space in the vicinity of the symmetry plane between the solenoids [2]. A profile of any particular force field must be excited by means of appropriate distribution of current density in the coils.

Superconducting magnets are expensive and as much information as possible about the performance of a separator is required in the initial stages of development. Apart from insufficient theoretical findings, preliminary experiments seemed very promising. Relevant philosophy of the development has been discussed in detail in [14].

Experiments were carried out with the iron-pole DRDS. Synthetic martite was employed as a well-defined ferromagnetic feed, and extrapolation procedure was established. The partition curves for martite correspond well with those found for natural minerals, which fact increased confidence in feed-independent operation of DRDS. Background of the scale- up is very simple. Assuming a constant saturation magnetization in the model experiments, and assuming ideal paramagnetics with constant magnetic susceptibility in the scaled- up separator, the following transformation is applicable [5]:

$$
(\beta / \rho) M_{s} \operatorname{grad} B=\frac{\chi B^{*} \operatorname{grad} B^{*}}{\mu_{0}}
$$

$B$ is the measured flux density in the iron-pole DRDS and $B^{*}$ is the corresponding superconducting split-coil flux density. These are obviously substantially different magnetic fields.

Several design studies have been carried out. Preliminary conservative approach was based on the isodynamic mode ii. The force density $\mathrm{B}^{*}$ gradB ${ }^{*}$ of $60 \mathrm{~T}^{2} / \mathrm{m}$ was found by a trial and error calculation of the coil [2]. This machine can be appropriate, for instance, for separation of Styrian siderite from ankerite gangue [14]. 
The trial-and-error design was not, however, found satisfactory. A basis for a straightforward method was first developed by Schmidt [10]. Later on, relevant computer programs were implemented by Hackhofer and Sammer $[15,16,17]$. Using this method, a superconducting isodynamic DRDS separator with force density up to $150 \mathrm{~T}^{2} / \mathrm{m}$ is within reach when using common $\mathrm{NbTi}$ superconductors.

Straightforward search for appropriate coil configurations is based on the required $\left|\mathrm{B}^{*}\right|$ profile over $\mathrm{r}$ along the symmetry plane of the separation space. $\left|\mathrm{B}^{*}\right|$ is derived from the forces, see eq. (2). This $\left|\mathrm{B}^{\star}\right|$ - profile is first approximated by an analytical function which is compatible with Maxwell equations [15]. Then the field outside the symmetry plane can be calculated with INFCAL (INverse Field CALculation), irrespective of any particular realization. Finally, boundary values for the channel or just outside the channel are defined. There is no need to calculate the field far away from the channel.

This particular field must be excited by the current conductors outside the separation space. CUMMAFD (CUrrent Minimum MAgnetic Field Design) allows an optimized approximation of the theoretical field defined by the boundaries $[15,16,17]$. The highest force densities can be found by maintaining individual currents in the winding. This is due to the limitations of the critical current of a superconductor. Critical currents depend strongly on the applied field.

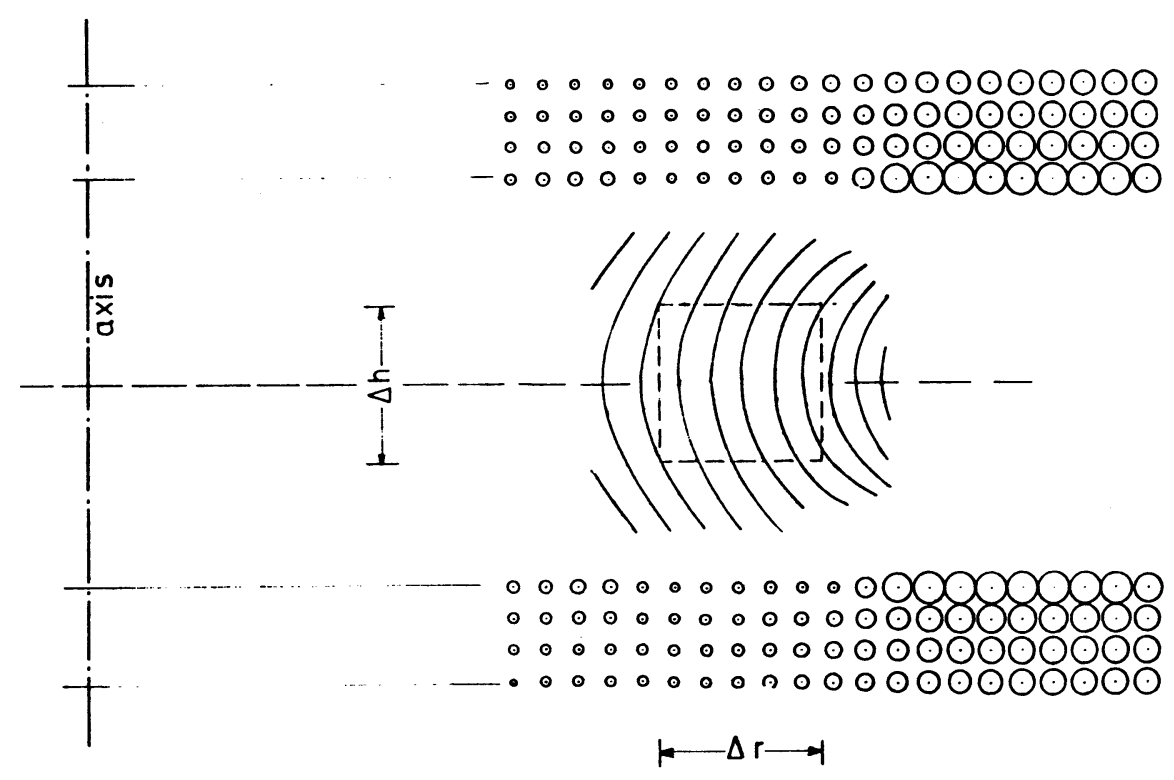

FIGURE 11a Superconducting split-coil system for DRDS: individual current distribution and $\mathrm{B}^{2}$-isolines across separation channel 
An example of recent design is shown in Figure 11 [5]. It is based on genuine neutral equilibrium hybrid mode iii., by taking into consideration experimental results, as well as the selectivity potential when adjusting tangential feed properly. Relative individual currents in the coils are indicated by respective circle diameters. $\mathrm{B}^{*}$ grad $\mathrm{B}^{*}$ can be determined from the distance between the $\mathrm{B}^{{ }^{2}}$ lines. Again, the forces are not strictly parallel. At the mean radius $\bar{r}$ of the channel the level of the force density can be seen from Fig. 11. It is clear that the force density of $150 \mathrm{~T}^{2} / \mathrm{m}$ is within reach with industrial $\mathrm{NbTi}$ superconductor. If $\mathrm{Nb}_{3} \mathrm{Sn}$ is used, the force density exceeding $280 \mathrm{~T}^{2} / \mathrm{m}$ can be obtained. However, the magnet size is limited and there is little chance to build single DRDS units much larger than that shown in the Figure.

The outlined method of design of a superconducting magnet is not limited to solenoids only. INFCAL and CUMMAFD can also be applied to various configurations whenever the magnetic vector potential has only one spatial component. Representative examples can be found in the literature $[5,15,16,17]$.

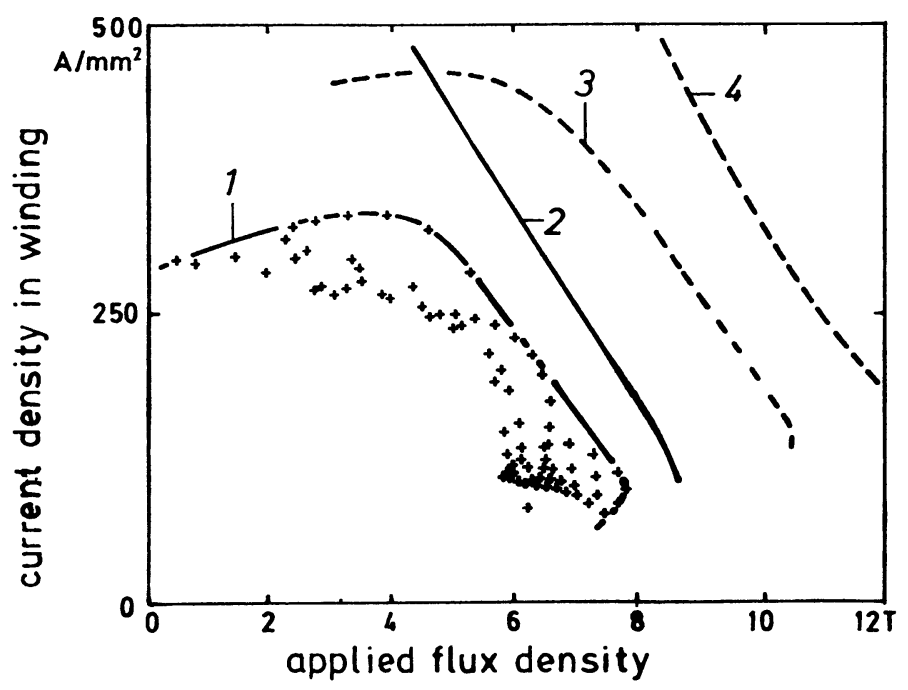

FIGURE 11b Superconducting split-coil system for DRDS: current density versus flux density chart. + - individual conductors, 1-envelope of individual conductors with $\mathrm{r}=205 \mathrm{~mm}, \mathrm{BgradB}=150$ $\mathrm{T}^{2} / \mathrm{m}$, 2-typical $\mathrm{NbTi}$ characteristics at $4.2 \mathrm{~K}$, 3-envelope of individual conductors with $\bar{r}=205 \mathrm{~mm}, \operatorname{BgradB}=280 \mathrm{~T}^{2} / \mathrm{m}$, 4-typical $\mathrm{Nb}_{3} \mathrm{Sn}$ characteristics at $4.2 \mathrm{~K}$ 
However, adequate winding techniques still have to be elaborated to make full use of benefits of the method. It transpires clearly that the most economical excitation of a distinct nonuniform magnetic field should be effected with a matching nonuniform current distribution in the coils rather than with a blocked coil system.

\section{OPERATIONAL DOMAIN AND COST ESTIMATION}

Figure 12 depicts the DRDS operational domains in terms of mass magnetic susceptibility. Iron-pole separators are intended for partly ferromagnetic feed materials; the lowest ferromagnetic volumetric content being of the order of $10 \%$ of the magnetics discharge. Superconducting machines can be useful for paramagnetic materials, with magnetic susceptibility as low as $10^{-7} \mathrm{~m}^{3} / \mathrm{kg}$.

A cost estimation for machines in the ferromagnetic feed domain can be easily shown. According to experience with existing iron-pole separators, investment of approximately $\$ 0.5$ per tonne of feed material is determined. Lifetime of a separator is assumed to be 10 years. Running costs are determined mainly by the energy consumption when electromagnets are used, i.e. approximately $\$ 1-2$ per tonne [9]. Permanent magnets may reduce this figure considerably. However, the setting of the separator is then possible only by adjusting the Froude number.

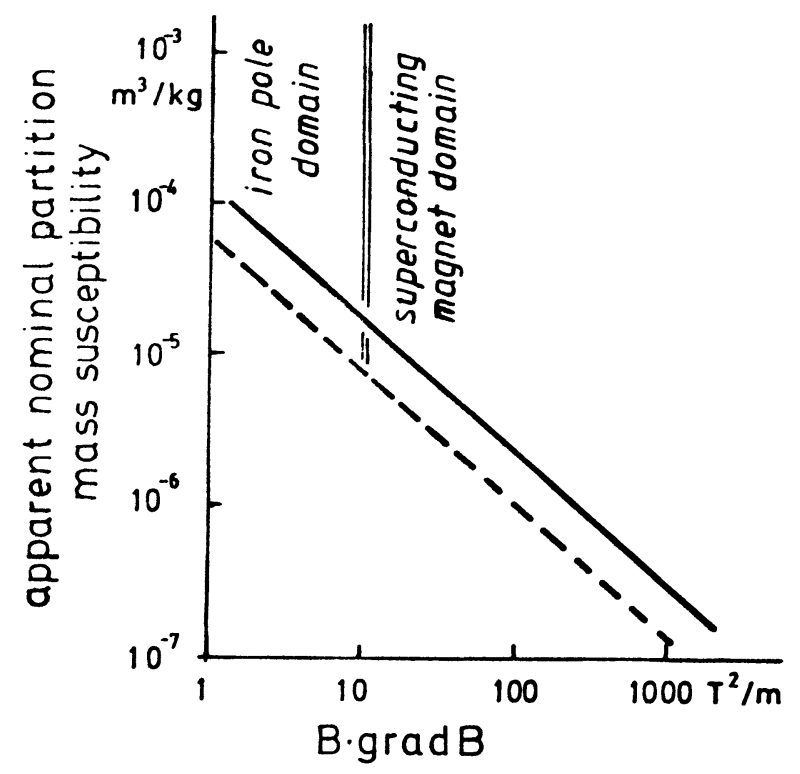

FIGURE 12 Domains of DRDS operation. ---- $Z=5 \times$ gravity, $-Z=10 x$ gravity 
The situation is much less favourable for a superconducting plant where the investment can approach $\$ 1$ million. This figure is prohibitive for a pilot plant, irrespective of the expected improvement in selectivity. Such a costly plant can be justified for very high quality products only, Simple primary ore beneficiation, where the separation cost must be low, it is not viable. Recycling or final processing in the course of multistage flowsheet can, however, be of interest for selective superconducting DRDS separators in the future.

\section{ACKNOWLEDGMENTS}

Development of DRDS was carried out in cooperation with ELINEnergieanwendung, Vienna. Valuable advice was provided by the University of Mining and Metallurgy, Leoben. Support of the Austrian Forschungsforderungsfonds der gewerblichen wirtschaft, the Fonds zur Forderung der wissenschaftlichen Forschung, and the Joanneum Research is greatly appreciated. The author is indebted to S. Grundorfer for critical reading of the manuscript.

\section{REFERENCES}

1. S.G. Frantz: US-Patent $2,056,426$ (1936)

2. J. Gerhold, J. Schmidt, S. Grundorfer and H. Fillunger, in Proceedings of MT9, Zurich (1985), p. 302

3. K. Lileg and B. Schnizer, to be published in IEEE Trans. Mag. MAG-27 (1992)

4. J. Svoboda, Magnetic Methods for the Treatment of Minerals (Elsevier, Amsterdam, 1987)

5. J. Gerhold, MT12, Leningrad (1991), to be published in IEEE Trans. Mag. MAG-27 (1992)

6. J. Gerhold and J. Schmidt, Proc. 4th World Filtration Congress, Ostend (1986), p. 3.9

7. J. Schmidt, Particle trajectories in a dry rotating disk separator, Internal Report (1986)

8. J. Gerhold, J. Schmidt, M. Hubmann, S. Grundorfer and H. Fillunger, IEEE Trans. Mag. MAG-24 (1988), 870 
9. H.J. Steiner, Meeting on magnetic separation, ELIN-Vienna 1989)

10. J. Schmidt, Systematic calculation of superconducting coils for specified magnetic field profiles. Doktorate Thesis, Technical University Graz (1987)

11. J. Sammer et al., IEEE Trans. Mag. MAG-26 (1990), 2064

12. J. Gerhold et al., Ind. Miner. Mines Carr. Tech. 73 (1991), 177

13. J. Gerhold, AT-Patent 390897 (1990)

14. J. Gerhold et al., XVIIth Intl. Congress on Refrigeration, Vol. A, Vienna (1987), p. 19

15. W. Hackhofer, Computation of air-cored coil arrangement for superconducting magnet separation. Doktorate Thesis, Technical University Graz (1990)

16. W. Hackhofer et al., JMMM 83 (1990), 493

17. J. Gerhold and W. Hackhofer, IEEE Trans. Mag. MAG-26 (1990), 1771

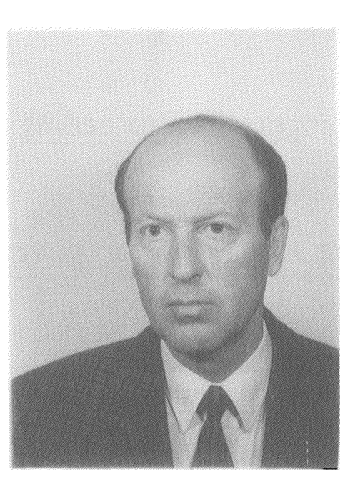

Juergen Gerhold received his M.Eng. degree in 1964 and his Dr.Eng. degree in 1970 in Electrical engineering from the Technical University in Graz, Austria. Until 1981 he was involved in field tests of a superconducting cable under the leadership of the late professor Klaudy. Presently he is a titular Professor and Head of the Cryogenic Department at the Institute for Electromagnetic Energy Conversion at Graz Technical University. His current interests include superconducting power equipment and magnetic separation technology.

Keywords: dry magnetic separation, selectivity, deflection mode of separation, ironpole magnets, superconducting solenoid magnets. 